\title{
Off-resonance Magnetisation Transfer Contrast (MTC) MRI using Fast Field-Cycling (FFC)
}

\author{
Chang-Hoon Choi, Gareth R. Davies, and David J. Lurie \\ Aberdeen Biomedical Imaging Centre, University of Aberdeen, Foresterhill, AB25 2ZD, \\ Aberdeen, Scotland, UK
}

Correspondence to:

Chang-Hoon Choi

Biomedical Physics, Aberdeen Biomedical Imaging Centre,

University of Aberdeen, Foresterhill, AB25 2ZD, Scotland, UK

Tel: +44 (0) 1224 553489, Fax: +44 (0) 1224552514

E-mail: changhoon.choi@abdn.ac.uk 


\section{Abstract}

Magnetisation transfer contrast (MTC) is an important MR contrast generating mechanism to characterise the undetectable bound protons indirectly using the decreased signal intensity of the observable free protons. MTC imaging typically employs a range of off-resonance RF pre-saturation pulse with maintaining the RF magnetic field $\left(B_{1}\right)$ at a specified value. However, this presents a technical difficulty, particularly at low field, because the larger offset frequencies tend to be outside the bandwidth of the RF transmit system, causing $B_{1}$ to vary with the frequency offset. Here, we demonstrate a novel off-resonance irradiation method using fast field-cycling which allows switching of the external magnetic field between several chosen strengths, while holding constant the RF frequency and $B_{1}$ level. This permits one to avoid the problem of $B_{1}$ variation as a function of frequency offset. The results obtained by this new technique are in excellent agreement with those obtained by the conventional technique.

Key words: Field cycling; Off-resonance; Magnetization Transfer; MTR; Z-spectra 


\section{Introduction}

The use of fast field-cycling (FFC) with MRI has increased over recent years. The FFC technique enables switching of the external magnetic field strength $\left(B_{0}\right)$, rapidly, between several discrete levels. This affords access to a number of new MR contrast mechanisms, for example, proton electron double resonance imaging [1], MR relaxometric imaging [2-4] and delta relaxation enhanced MR [5]. In this work, we have applied the FFC technique to magnetisation transfer contrast (MTC) MRI [6].

MTC has been established as one of the important mechanisms for manipulating tissue contrast in MRI [7] and this has led to its application in numerous clinical areas [8-10]. The simplest model of MTC considers the protons in two "pools", namely the free pool and the bound pool [11,12]. In biological tissue, the free protons (e.g., in water) have a narrow line-width (about $50 \mathrm{~Hz}$ ) and give a high signal intensity. In comparison, the bound protons (e.g., in macromolecules) which have a broad line-width (about $50 \mathrm{kHz}$ ) give rise to a nearzero signal intensity. MTC utilises the difference in line-widths by applying, in its simplest implementation, a continuous-wave off-resonance irradiation pulse (or "MT pulse") to saturate the bound protons, with high saturating efficiency, but with very low direct saturation of the free protons $[12,13]$.

In order to obtain useful data from an MTC experiment, it is important to measure the MT effect at a range of RF offset frequencies while maintaining the RF magnetic field strength $\left(B_{1}\right)$ at predetermined amplitude. However, $B_{1}$ is likely to decrease significantly with increasing RF offset frequency, particularly at low field, because of the limited bandwidth of the RF transmit system. Thus, $B_{1}$ calibration at each of the discrete RF offset frequencies becomes necessary. Furthermore, the available range of offset frequencies may also be limited.

Here, we demonstrate an alternative off-resonance MTC method using the FFC technique which makes it possible to counter these complications. The MT pulse is always applied at zero RF offset frequency with constant RF amplitude (consequently, constant $B_{1}$ ) while $B_{0}$ is adjusted through FFC in order to achieve off-resonance irradiation. This provides the same off-resonance effect as does the conventional MTC method, without the necessity for $B_{1}$ re-calibration, and with effectively no limitation on the RF offset (since it is determined by the range of the magnetic field-cycling). 


\section{Methods}

All experiments were carried out using a home-built field-cycling MRI system [1] that uses a permanent magnet for detection and a co-axial resistive magnet for field-offset. The operating field strength of the permanent magnet is fixed at $58.7 \mathrm{mT}$ (corresponding to 2.499 $\mathrm{MHz}$ proton Larmor frequency). However, the effective $B_{0}$ can be set at any value from zero to approximately $100 \mathrm{mT}$ through field compensation by the resistive magnet. A shielded split-solenoid (nine turns each) NMR transmit/receive RF coil was used with length $100 \mathrm{~mm}$, diameter $75 \mathrm{~mm}$, and an unloaded $Q$-factor of 471. A home-made RF amplifier tailored to suit the continuous-wave MTC experiments was used with a peak power of approximately $100 \mathrm{~W}$. The pulse sequences to control these experiments were written using a C-like programming language on a commercial console (SMIS Ltd., UK).

$1 \%, 2 \%$, and $4 \%(\mathrm{w} / \mathrm{v})$ agarose gel (Sigma-Aldrich, UK) samples were prepared either with distilled water or with $0.04 \mathrm{mM} \mathrm{MnCl} 2$ solution. This small amount of $\mathrm{MnCl}_{2}$ significantly influences $T_{1}$ but not $T_{2}$. For example, $T_{1}$ values of the $2 \%$ agarose gel with and without $\mathrm{MnCl}_{2}$ were $967 \mathrm{~ms}$ and $1161 \mathrm{~ms}$, respectively, whilst $T_{2}$ values were $63 \mathrm{~ms}$ and 64 ms. In addition, a sample only containing aqueous solution of $\mathrm{MnCl}_{2}$ (without agarose) was used as a control. A phantom constructed from the seven samples was used for imaging experiments.

Fig. 1 illustrates the differences between the irradiation method used in conventional MTC and the novel FFC off-resonance irradiation method. In the conventional method (Fig. 1a), $B_{0}$ is constant throughout the MR scan. In the FFC method (Fig.1b), $B_{0}$ switches to a predetermined level (usually referred to as the evolution magnetic field, $B_{0 \mathrm{e}}$ ). The MT saturation pulse is then applied, for a set duration, before $B_{0}$ returns to its original level for MR data acquisition. Moreover, Fig. 1a illustrates the required RF amplitude with varying $\mathrm{RF}$ offset frequency for a given $B_{1}$; larger RF amplitude is required at a higher offset frequency than at a lower offset frequency in order to compensate for reduced efficiency of the RF transmit chain at the extremities of its bandwidth. Therefore, it is essential to measure the required RF amplitudes as a function of RF offset frequency for a desired $B_{1}$. Alternatively, in the new technique (Fig. 1b), the RF amplitude and the offset frequency are fixed at a specified value but $B_{0}$ is precisely adjusted. For example, in order to achieve an effective $11 \mathrm{kHz}$ offset frequency irradiation, the MT pulse remains at a zero offset (2.499 $\mathrm{MHz}$ ) but the applied evolution field is set to $58.44 \mathrm{mT}$ (proton Larmor frequency 2.488 $\mathrm{MHz}$ ). The difference in the MT effect between these two field strengths will be negligible, 
since the $T_{1}$ values of the samples are identical (within experimental error) when measured at 2.488 MHz and 2.499 MHz. The magnetic field is switched between levels within $5 \mathrm{~ms}$. However, a delay of approximately $60 \mathrm{~ms}$ is required to ensure field stabilisation before applying the 90 degree pulse for MR data acquisition.

Since the MT effect is strongly related to the MT pulse parameters (the duration of MT irradiation, $B_{1}$, and the offset frequency) $[11,14,15]$, the MT pulse duration which attains the steady state condition was initially determined. With this duration determined, the MT effects as a function of the offset frequency with varying $B_{1}$ were studied. In this study, 32 offset frequencies were chosen from 0.01 to $49 \mathrm{kHz}$ and seven $B_{1}$ values $(5,7.5,10,15,20$, 30 , and $40 \mu \mathrm{T}$ ) were used. The offset frequencies were uniformly distributed on a logarithmic scale in order to cover a wide range of offsets, with higher frequency-resolution close to resonance (zero to $1 \mathrm{kHz}$ offset range). The RF amplitudes of the MT pulses were measured as a function of the RF offset frequency using a search coil and an oscilloscope. $B_{1}$ calibration curves were then obtained and the results were incorporated into the pulse sequences to conduct the conventional off-resonance MTC experiments. As expected, the calibration curves depended on the choice of RF coil and RF amplifier.

Fig. 2 shows a set of experimentally determined calibration curves, obtained using the split-solenoid RF coil and the home-built amplifier. The required amplitudes significantly increase at higher offset frequencies. Thereafter, the relationships between each MT pulse parameter and the MT effect were analysed and the conditions required to achieve the maximum MT effect with each sample were determined.

The MT effects measured by both techniques were compared through the evaluation of the z-spectra [16] and the magnetisation transfer ratios (MTRs) [17] which are common tools to estimate or quantify the MT effect. The z-spectra show normalised longitudinal magnetisation of the free protons with and without MT pulse irradiation over a range of MT irradiation offset frequencies. Thus, they provide important insights for interpreting the MT effects occurring in both pools. Four images of a phantom were acquired with an interleaved (with and without irradiating MT pulse) gradient echo imaging pulse sequence using the two different methods (with frequency switching (conventional) or with fast field-cycling). In order to assess the MT effects, manually segmented regions of interest within the images were drawn and MTR values of each sample were calculated according to the standard equation MTR $=\left(M_{0}-M_{s}\right) / M_{s}$, with $M_{s}$ and $M_{0}$ being the signal intensities of the free protons measured with and without the off-resonance irradiation, respectively. The pixel-by-pixel 
based MTR images were also obtained by image processing using MATLAB (MathWorks, Natick, MA, USA).

\section{Results}

Using the conventional off-resonance irradiation method, the MT effect was observed as a function of the MT pulse duration (from $0 \mathrm{~s}$ to $5 \mathrm{~s}$ in $0.1 \mathrm{~s}$ increment) in order to ensure that a steady state had been reached. All the samples approached the steady state within the MT pulse duration of $4 \mathrm{~s}$. The MT effect was also measured as a function of offset frequency for a number of $B_{1}$ values as shown in Fig. 3a. The curves with solid markers describe the normalised magnetisation $\left(\mathrm{M}_{\mathrm{s}} / \mathrm{M}_{0}\right)$ observed from a $4 \%$ agarose gel sample. Since the maximum observed MT effect occurs with minimum direct saturation of the free protons (called the direct effect), the estimation of the direct effect (the unfilled markers in Fig. 3a) was generated using the equation given by Eng et al. [11]. From the above, the conditions for the maximum MT effect with each sample were determined by subtracting the calculated direct effect $\left(\mathrm{M}_{\mathrm{sdir}} / \mathrm{M}_{0}\right)$ from the observed MT effect $\left(\mathrm{M}_{\mathrm{s}} / \mathrm{M}_{0}\right)$ (Fig. 3b). The combination of $B_{1}$ of $25 \mu \mathrm{T}$ and an offset frequency of $11 \mathrm{kHz}$, typically, produced a close to maximal MT effect for each sample.

Using the above MT pulse conditions, z-spectra of $1 \%, 2 \%$ and $4 \%$ agarose gels collected by the conventional RF offset method were compared with z-spectra obtained by the novel FFC method. Results of the comparison are shown in Fig.4, where it can be seen that the results from the two methods were in close agreement.

A multi-sample phantom was imaged using both off-resonance irradiation techniques, as illustrated in Fig. 5a. The samples A, B, and $\mathrm{C}$ were 1\%, 2\%, and 4\% agarose gels respectively made up with distilled water. The samples A', B', and C' were 1\%, $2 \%$, and 4\% agarose gels respectively made up with distilled water containing $0.04 \mathrm{mM} \mathrm{MnCl}_{2}$. The control sample $\left(0.04 \mathrm{mM} \mathrm{MnCl}_{2}\right.$ solution) was located at the centre of the phantom. The imaging parameters were: $10 \mathrm{~s}$ repetition time, $20 \mathrm{~ms}$ echo time, $120 \mathrm{~mm}^{2}$ field of view, 128 pixel by 128 pixel image matrix size, $15 \mathrm{~mm}$ slice thickness, NEX $=4,25 \mu \mathrm{T} B_{1}, 11 \mathrm{kHz}$ offset (or $58.44 \mathrm{mT} B_{0 \mathrm{e}}$ ), and $4 \mathrm{~s} \mathrm{MT}$ pulse duration. These parameters were set to be the same for all experiments using both techniques. The top row images in Fig 5a were obtained from the new FFC technique with (image 2) and without (image 1) MT irradiation whereas the bottom row images ( 1 ' and 2') were obtained from the conventional RF offset technique. Moreover, the intensity of images ( 3 and 3') is proportional to the MTR values obtained from 
the relevant data sets from images 1 and 2 (1' and 2') after processing with MATLAB. Fig. $5 \mathrm{~b}$ shows the quantified MTR values using the selected regions of interest from the images of the individual samples. Due to the absence of macromolecules in the control sample, the MT effect is almost zero. Only a very small direct effect is present. In contrast, the MT effects of the other samples increase with increasing concentration of agarose. Furthermore, smaller MT effects are observed in the samples doped with $\mathrm{MnCl}_{2}$ because of their shorter $T_{1}$ values [18]. Above all, these results prove an excellent agreement (within 3\%) between the measurements collected by the FFC-MTC and the RF-MTC methods.

\section{Discussion}

We have demonstrated the applicability of a new off-resonance technique for MTC MRI. The main obstacle in the use of the conventional off-resonance RF-MTC technique at ultra-low field is the necessity for $B_{1}$ re-calibration over a range of offset frequencies. With the new FFC technique, this obstacle is bypassed through switching $B_{0}$ in the place of the RF offset frequency. As a result, there is no need to re-calibrate the RF amplitude at each offset frequency. The comparison of data, for the z-spectra and the MTR values, obtained by the new method with that from the conventional method demonstrated a convincing agreement in the results of these two techniques. As expected, the results showed that the MT effect was more pronounced in the samples with higher macromolecule (agarose) content. It was also shown that the MT effects were diminished in the samples of agarose gels containing paramagnetic substances (smaller MTR values).

However, we found that a certain extent of the MT effect seemed to be reduced when the new technique was used. The loss of the MT effect was larger at near zero offset frequency and at higher concentration of agarose in the sample. It can be attributed to the recovery of longitudinal magnetisation during the delay required for the magnetic field stabilisation. Nevertheless, the loss was not significant at the $11 \mathrm{kHz}$ offset where the maximum MT effect occurred. The current system has the limitation of a maximum field changing rate and thus minimum ramp times for a typical MT experiment of $5 \mathrm{~ms}$, followed by field-settling time of approximately $60 \mathrm{~ms}$. Our next generation of fast field-cycling MR imager currently under construction in our laboratory will overcome these drawbacks by decreasing these combined delays.

Ceckler and Balaban previously demonstrated the $B_{0}$ dependence of the MT effect, which was particularly significant around the $30 \mathrm{MHz}$ proton Larmor frequency range [19]. 
Using our present apparatus (as used in this study), we are currently embarking on experiments to explore the Larmor frequency dependence of the MT effect over the range up to $2.5 \mathrm{MHz}$. Furthermore, the next-generation instrument will have a detection field of $0.5 \mathrm{~T}$, so will allow measurements in the $20 \mathrm{MHz}$ range, where there may be significant interest in the $B_{0}$ dependence of the MT effect.

The interest in using chemical exchange saturation transfer (CEST) or paramagnetic CEST (PARACEST) technique has also increased [20,21]. In common with MTC, an offresonance saturation pulse is applied in experiments using the CEST or the PARACEST techniques. Through irradiation, CEST protons are saturated and then detected indirectly via the reduced free water signal. Our new off-resonance FFC technique has the potential for use in the measurement of CEST and PARACEST effects.

At present, MR scanners with field-cycling capability are rather scarce, and are limited to home-built systems. However, Alford et al. have recently shown that field-cycling can be achieved on a commercial, superconducting-magnet, clinical MR scanner, using a field-offset insert coil which is placed into the bore of the scanner [5, 22]. In this way fieldcycling, and MTC with field cycling, could become available to the wider MRI community.

There is currently a dearth of experimental data concerning measurements of MT parameters as a function of field strength, at low magnetic field. The method presented here offers an efficient way of making these measurements, which could shed extra light on the fundamental mechanisms of MTC.

\section{Acknowledgements}

The authors acknowledge financial support for the FFC-MRI project from Research Councils UK and the Engineering and Physical Sciences Research Council, under the Basic Technology scheme (grant number EP/E036775/1). 


\section{References}

[1] D.J. Lurie, M.A. Foster, D. Yeung, J.M.S. Hutchison, Design, construction and use of a large-sample field-cycled PEDRI imager, Phys. Med. Biol. 43 (7) (1998) 1877-1886.

[2] J.W. Carlson, D.M. Goldhaber, A. Brito, L. Kaufman, MR relaxometry imaging. Work in progress, Radiology 184 (1992) 635-639.

[3] D.J. Lurie, Quadrupole-dips measured by whole-body field-cycling relaxometry and imaging, In Proceedings of the $7^{\text {th }}$ Annual Meeting of ISMRM, Philadelphia, USA (1999) p.653.

[4] S.E. Ungersma, N.I. Matter, J.W. Hardy, R.D. Venook, A. Macovski, S.M. Conolly, G.C. Scott, Magnetic resonance imaging with $T_{1}$ dispersion contrast, Magn. Reson. Med. 55 (6) (2006) 1362-1371.

[5] J.K. Alford, B.K. Rutt, T.J. Scholl, W.B. Handler, B.A. Chronik, Delta relaxation enhanced MR: Improving activation-specificity of molecular probes through $R_{1}$ dispersion imaging, Magn. Reson. Med. 61 (4) (2009) 796-802.

[6] C-H. Choi, G.R. Davies, D.J. Lurie, Off-resonance magnetisation transfer contrast MRI using fast field-cycling technique, In Proceedings of the $17^{\text {th }}$ Annual Meeting of ISMRM, Honolulu, USA (2009) p.2747.

[7] S.D. Wolff, R.S. Balaban, Magnetization transfer contrast (MTC) and tissue water proton relaxation in vivo, Magn. Reson. Med. 10 (1) (1989) 135-144.

[8] A. Boss, P. Martirosian, K. Kuper, G. Fierlbeck, C.D. Claussen, F. Schick, Whole-body magnetization transfer contrast imaging, J. Magn. Reson. Imaging 24 (5) (2006) 1183-1187.

[9] R.R. Edelman, S.S. Ahn, D. Chien, W. Li, A. Goldmann, M. Mantello, J. Kramer, J. Kleefield, Improved time-of-flight MR angiography of the brain with magnetization transfer contrast, Radiology 184 (2) (1992) 395-359.

[10] M. Filippi, Magnetization transfer MRI in multiple sclerosis and other central nervous system disorders, Eur. J. Neurol. 10 (1) (2003) 3-10.

[11] J. Eng, T.L. Ceckler, R.S. Balaban, Quantitative 1H magnetization transfer imaging in vivo, Magn. Reson. Med. 17 (2) (1991) 304-314. 
[12] R.M. Henkelman, X. Huang, Q-S. Xiang, G.J. Stanisz, S.D. Swanson, M.J. Bronskill, Quantitative interpretation of magnetization transfer, Magn. Reson. Med. 29 (6) (1993) 759766.

[13] J. Hua, G.C. Hurst, Analysis of on- and off-resonance magnetization transfer techniques, J. Magn. Reson. Imaging 5 (1) (1995) 113-120.

[14] P.T. Niemi, M.E.S. Komu, S.K. Koskinen, Tissue specificity of low-field-strength magnetization transfer contrast imaging, J. Magn. Reson. Imaging 2 (2) (1992) 197-201.

[15] W. Kucharczyk, P.M. Macdonald, G.J. Stanisz, R.M. Henkelman, Relaxivity and magnetization transfer of white matter lipids at MR imaging: Importance of cerebrosides and pH, Radiology 192 (2) (1994) 521-529.

[16] J. Grad, R.G. Bryant, Nuclear magnetic cross-relaxation spectroscopy, J. Magn. Reson. 90 (1) (1990) 1-8.

[17] R.M. Henkelman, G.J. Stanisz, S.J. Graham, Magnetization transfer in MRI: A review, NMR Biomed. 14 (2) (2001) 57-64.

[18] J.J. Tessier, N. Dillon, T.A. Carpenter, L.D. Hall, Interpretation of magnetization transfer and proton cross-relaxation spectra of biological tissues, J. Magn. Reson. Ser B. 107 (2) (1995) 138-144.

[19] T.L. Ceckler, R.S. Balaban, Field dispersion in water-macromolecular proton magnetization transfer, J. Magn. Reson. Ser B. 105 (1994) 242-248

[20] V. Guivel-Scharen, T. Sinnwell, S.D. Wolff, R.S. Balaban, Detection of proton chemical exchange between metabolites and water in biological tissues, J. Magn. Reson. 133 (1) (1998) 36-45.

[21] S. Zhang, M. Merritt, D.E. Woessner, R.E. Lenkinski, A.D. Sherry, PARACEST agents: Modulating MRI contrast via water proton exchange, Acc. Chem. Res. 36 (10) (2003) 783790.

[22] J.K. Alford, T.J. Scholl, W.B. Handler, B.A. Chronik, Design and construction of a prototype high-power $B_{0}$ insert coil for field-cycled imaging in superconducting MRI systems, Concepts Magn. Reson. B. 35 (1) (2009) 1-10. 

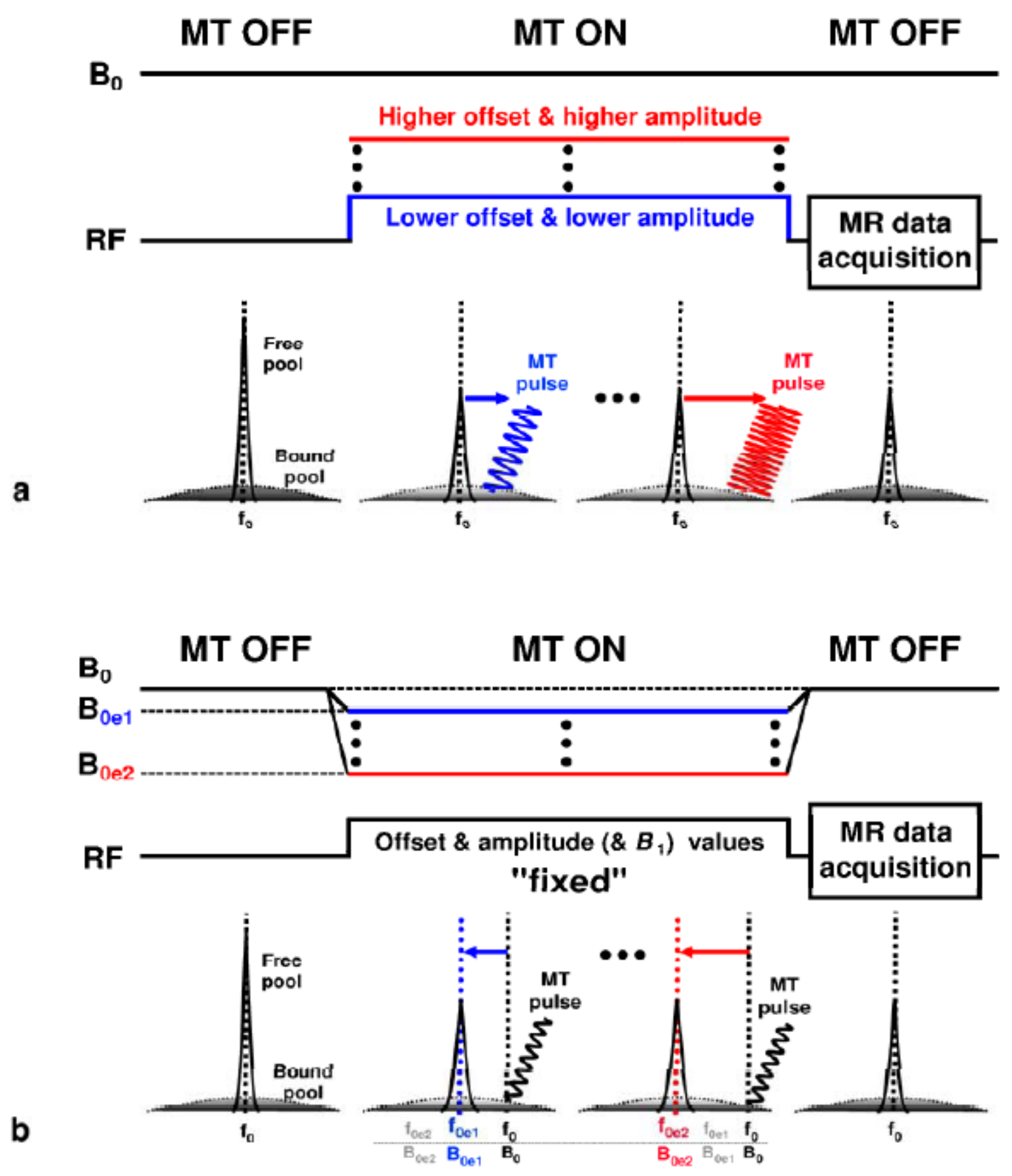

Fig. 1. Schematic comparison of (a) the conventional RF offset technique and (b) the novel FFC technique associated with the difference of pulse sequence timing diagrams (particularly, $B_{0}$ and RF pulse lines) and the status of both spin pools with and without off-resonance MT irradiation. 


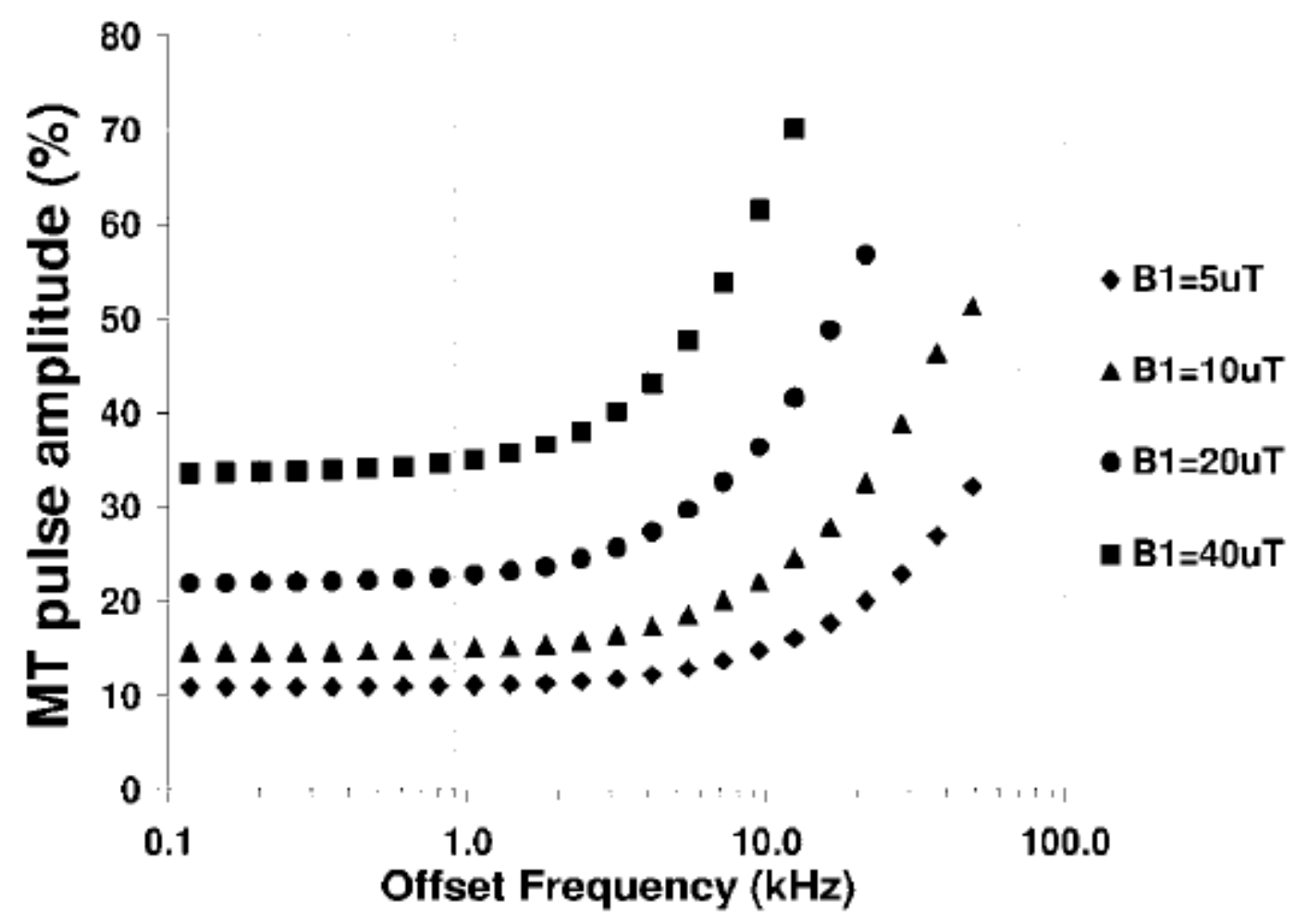

Fig. 2. $B_{1}$ calibration curves - the required MT pulse amplitude as a function of MT offset frequency for the desired $B_{1}(5,10,20$ and $40 \mu \mathrm{T})$. 

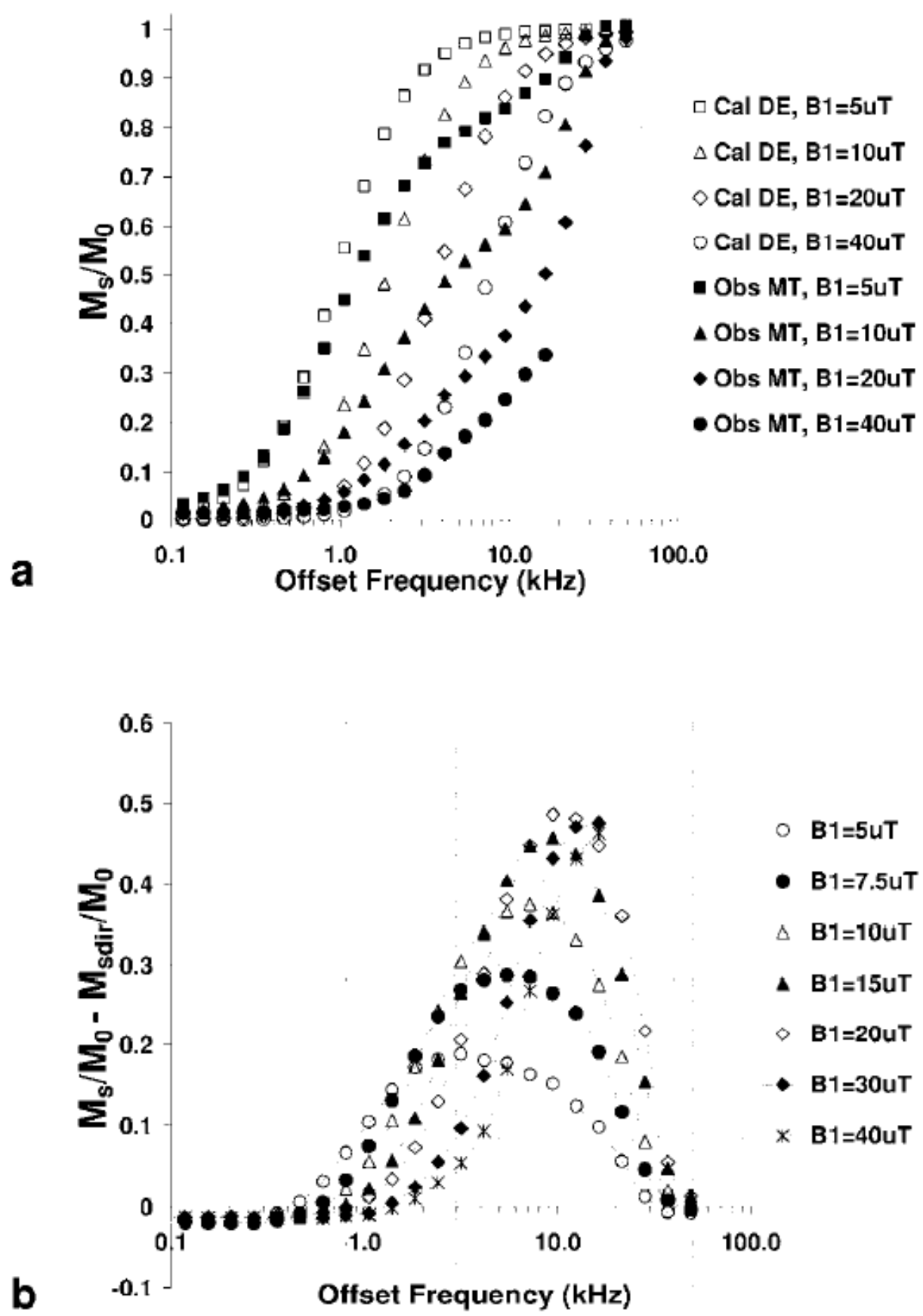

Fig. 3. (a) The normalised magnetisation $\left(M_{s} / M_{0}\right)$ of $4 \%$ agarose gel $\left(M_{s}\right.$ is the signal intensity of free protons with MT irradiation and $\mathrm{M}_{0}$ is without). The solid markers refer to the observed magnetisation transfer data while the unfilled markers refer to the calculated direct effects with different $B_{1}$ values. (b) The conditions required to maximise the MT effect of $4 \%$ agarose gel were determined by subtraction of the calculated direct effects (Cal DE) from the observed MT effects (Obs MT) for a range of offset frequencies and $B_{1}$ magnitudes. The combination of $25 \mu \mathrm{T} B_{1}$ and $11 \mathrm{kHz}$ frequency offset appears to be the optimum condition for MTC in this sample. 
a

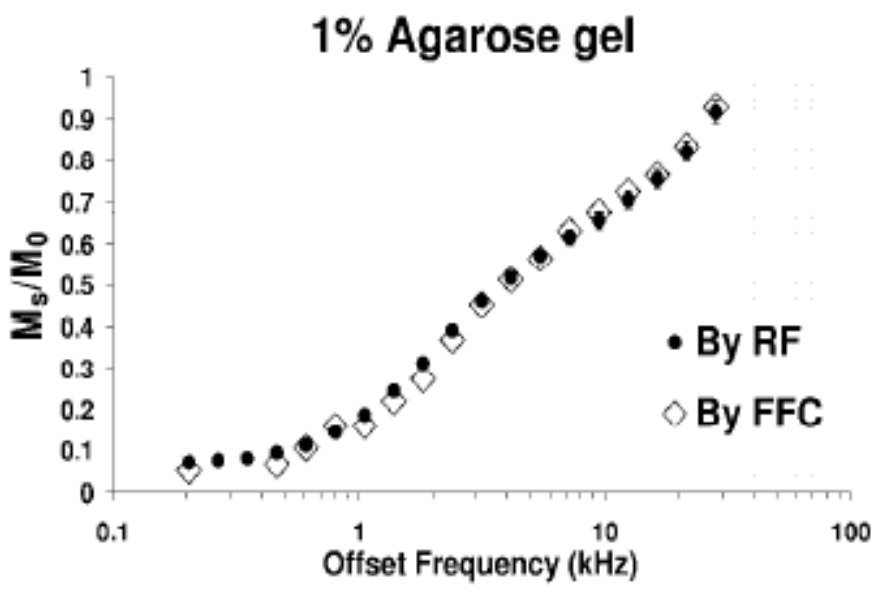

$2 \%$ Agarose gel

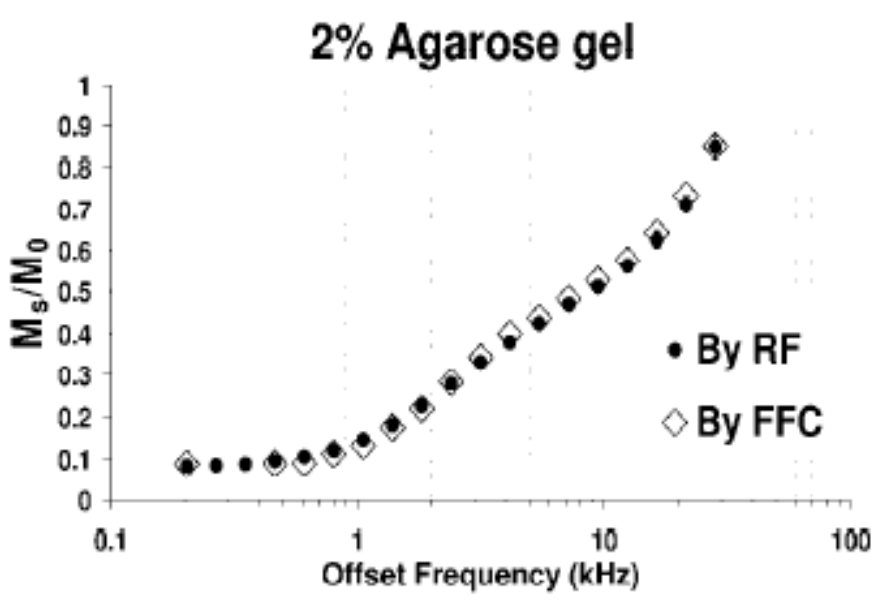

b

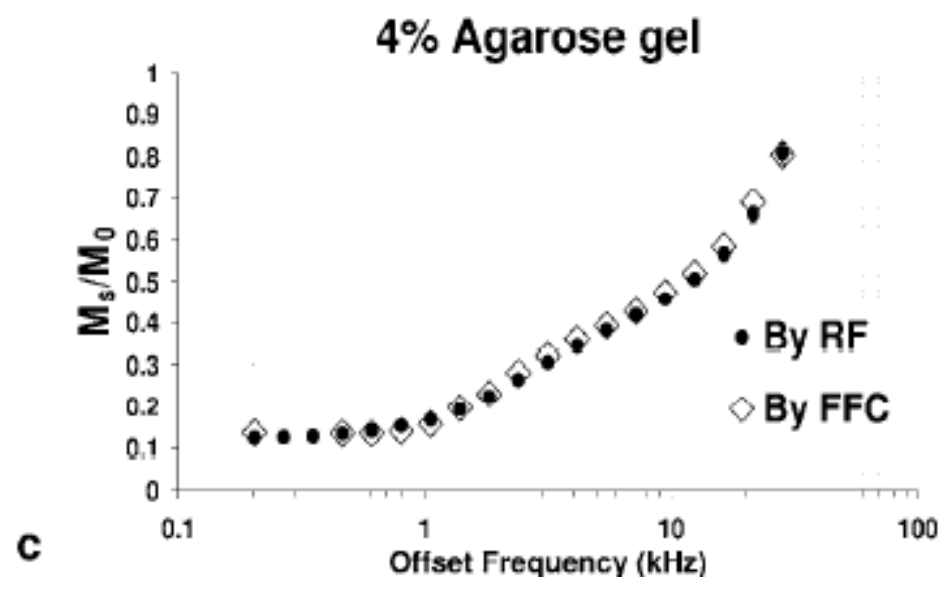

Fig. 4. Z-spectra of (a) 1\%, (b) 2\%, and (c) 4\% agarose gel samples obtained by the RF offset $(\bullet)$ and the FFC $(\diamond)$ techniques. 

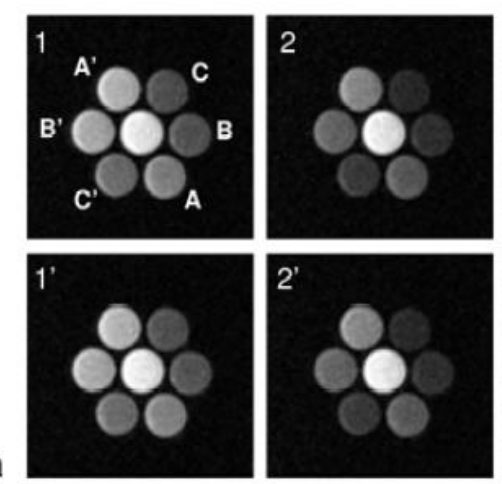
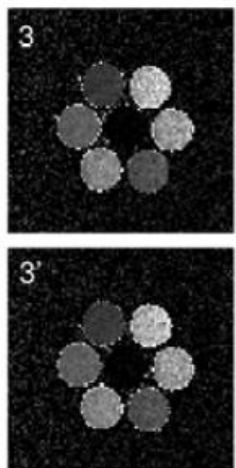

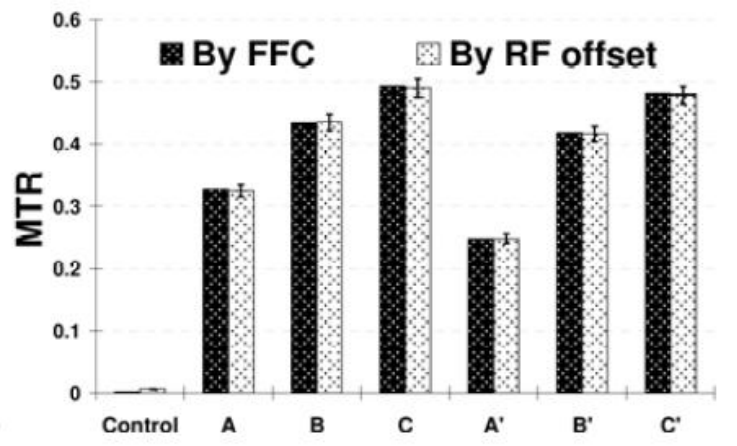

Fig. 5. (a) Experimental results obtained by conventional (bottom row) and new (top row) methods before (images 1 and 1') and after (images 2 and 2') MT irradiation and MTR images (3 and 3'), (b) Calculated MTR values for each component of the phantom derived from the RF offset and the FFC MTC techniques. 